\title{
A novel pore-region mutation, c.887G > A (p.G296D) in KCNQ4, causing hearing loss in a Chinese family with autosomal dominant non-syndromic deafness 2
}

Bangqing Huang ${ }^{1,2+}$, Yanping Liu ${ }^{3+}$, Xue Gao ${ }^{1,3}$, Jincao Xu ${ }^{3}$, Pu Dai ${ }^{1 *}$, Qingwen Zhu ${ }^{4^{*}}$ and Yongyi Yuan ${ }^{1 *}$

\begin{abstract}
Background: Hereditary non-syndromic hearing loss is the most common inherited sensory defect in humans. The KCNQ4 channel belongs to a family of potassium ion channels that play crucial roles in physiology and disease. Mutations in KCNQ4 underlie deafness non-syndromic autosomal dominant 2, a subtype of autosomal dominant, progressive, high-frequency hearing loss.

Methods: A six-generation Chinese family from Hebei Province with autosomal dominantly inherited, sensorineural, postlingual, progressive hearing loss was enrolled in this study. Mutation screening of 129 genes associated with hearing loss was performed in five family members by next-generation sequencing (NGS). We also carried out variant analysis on DNA from 531 Chinese individuals with normal hearing as controls.

Results: This family exhibits postlingual, progressive, symmetrical, bilateral, non-syndromic sensorineural hearing loss. NGS, bioinformatic analysis, and Sanger sequencing confirmed the co-segregation of a novel mutation [c.887G > A (p.G296D)] in KCNQ4 with the disease phenotype in this family. This mutation leads to a glycine-toaspartic acid substitution at position 296 in the pore region of the KCNQ4 channel. This mutation affects a highly conserved glutamic acid. NGS is a highly efficient tool for identifying gene mutations causing heritable disease.

Conclusions: Progressive hearing loss is common in individuals with KCNQ4 mutations. NGS together with Sanger sequencing confirmed that the five affected members of this Chinese family inherited a missense mutation, c.887G > A (p.G296D), in exon 6 of KCNQ4. Our results increase the number of identified KCNQ4 mutations.
\end{abstract}

Keywords: KCNQ4, Autosomal dominant non-syndromic deafness 2, Novel mutation, Next-generation sequencing

\section{Background}

Hearing loss is a very common sensory defect and a highly heterogeneous sensory disorder in humans. The majority of congenital cases of hearing loss are attributable to genetic factors. Hereditary hearing loss can be classified according to the pattern of inheritance, and

\footnotetext{
* Correspondence: daipu301@vip.sina.com; zqw301@163.com; yyymzh@163.com

'Equal contributors

'Department of Otolaryngology, Chinese PLA General Hospital, Beijing 100853, China

${ }^{4}$ Department of Otolaryngology, The Second Hospital Of Hebei Medical University, Shijiazhuang 050018, China

Full list of author information is available at the end of the article
}

the presence (syndromic) or absence (non-syndromic) of distinctive clinical features. The inheritance patterns of non-syndromic hearing loss (NSHL) include autosomal dominant, autosomal recessive, X-linked, and mitochondrial inheritance. Autosomal dominant deafness accounts for approximately $20 \%$ of cases of hereditary hearing loss [1]. Autosomal dominant non-syndromic hearing loss (ADNSHL) has extremely heterogeneous genetic and clinical features. To date, more than 60 loci for ADNSHL have been mapped to chromosomal regions and 27 genes for non-syndromic deafness, autosomal dominant (DFNA) have been identified (http:// hereditaryhearingloss.org). Recently, high-throughput

\section{() BioMed Central}

(c) The Author(s). 2017 Open Access This article is distributed under the terms of the Creative Commons Attribution 4.0 International License (http://creativecommons.org/licenses/by/4.0/, which permits unrestricted use, distribution, and reproduction in any medium, provided you give appropriate credit to the original author(s) and the source, provide a link to the Creative Commons license, and indicate if changes were made. The Creative Commons Public Domain Dedication waiver (http://creativecommons.org/publicdomain/zero/1.0/) applies to the data made available in this article, unless otherwise stated. 
sequencing, involving targeted sequencing of the protein-coding subset of the human genome, has become a highly efficient tool due to its ability to perform parallel sequencing of millions of nucleotides at relatively low cost and high speed. This should greatly improve the screening of thousands of target genes, making this an ideal technique for identifying causative genes and mutations involved in heritable hearing disease [2].

Based on the number of reported mutations, KCNQ4 (the gene responsible for DFNA2) is one of the genes most commonly responsible for ADNSHL [3]. KCNQ4 (voltage-gated potassium channel, KQT-like subfamily Q, member 4), the first identified causal gene of ADNSHL at the DFNA2 locus, was discovered and cloned by Kubisch in 1999 [4]. KCNQ4 was mapped to 1p34, within the DFNA2 locus; KCNQ4 is a member of the voltage-gated potassium channel family and plays a pivotal role in potassium recycling in the inner ear. Its cDNA encodes a polypeptide of 695 amino acids that forms a voltage-gated potassium Kv7.4 channel protein. $K C N Q 4$, together with $K C N Q 1, K C N Q 2$, and $K C N Q 3$, constitutes a distinct branch of the superfamily of voltage-gated channels [4].

These voltage-gated channels typically contain four subunits that encircle a central pore, which enables the selective passage of potassium ions across the cell membrane. Each subunit consists of six transmembrane segments (S1-S6) encoded by six exons (exons 2 to 7), with both $\mathrm{N}$ - and C-termini being located on the intracellular side of the membrane. The S4 segment comprises the voltage sensor of the channel; and the S5 and S6 portions, connected with an intervening re-entrant loop (P-loop domain), form the pore region. Four P-loop domains combine to form the selectivity filter of the channel [5]. KCNQ4 is expressed in sensory hair cells of the inner ear and in the central auditory pathway, defects in KCNQ4 caused hearing loss by a slow degeneration of outer hair cells resulting from chronic depolarization [6]. Autosomal dominant non-syndromic hearing loss causing by $\mathrm{KCNQ} 4$ mutations usually starts from high-frequency. Most of the missense mutations identified to date are located in the pore region of the KCNQ4 channel, namely, the P-loop domain [4]. Missense mutant in pore region, e.g. p.G285S and p.G296S, exerts a strong dominant-negative effect on potassium currents by reducing the wild type KCNQ4 channel expression at the cell surface, causing a greater reduction of KCNQ4 current to the cell membrane [4, 7]. In this study, we report the genetic basis of ADSHNL in a Chinese family, as determined by NGS together with Sanger sequencing, and identify a novel missense mutation, c.887G > A (p.G296D), in the pore region of the KCNQ4 channel.

\section{Methods}

\section{Family members and clinical evaluations}

The family, referred to here as HBJ, is a six-generation Chinese family with 35 members of Han origin from Hebei Province with autosomal dominant, postlingual, progressive, non-syndromic sensorineural hearing loss (Fig. 1). Eight members of this family participated in our study, including five affected, and three unaffected. Medical histories of the members of the family were obtained via a questionnaire on the following aspects of this condition: subjective degree of hearing loss (the clinical history ruled out environmental factors as the cause of hearing loss), age at onset, progression, symmetry of the hearing impairment, use of aminoglycosides, presence of tinnitus, use of hearing aids, noise exposure, medication, pathological changes in the ear, and other relevant clinical manifestations. Physical examinations ruled out the possibility of syndromic hearing loss. Audiometric evaluations and otological examinations included otoscopy, pure tone audiometry (PTA), acoustic immittance measurement, auditory brainstem responses, and distortion product otoacoustic emissions (DPOAE). PTA was calculated as the average of the thresholds measured at $0.5,1.0,2.0$, and $4.0 \mathrm{kHz}$, and performed to test for air conduction $(125-8000 \mathrm{~Hz})$ and bone conduction $(250-4000 \mathrm{~Hz})$. The severity of hearing impairment was defined as mild (26-40 dB), moderate $(41-55 \mathrm{~dB})$, moderately severe $(56-70 \mathrm{~dB})$, severe (71-90 dB), or profound ( $>90 \mathrm{~dB})$. Tympanometry indicated proper functioning of the middle ear. A high-resolution computed tomography (HRCT) scan of the temporal bone was performed on some of the affected individuals. The diagnosis of profound sensorineural hearing impairment was made in accordance with the ICD-10 (International Classification of Diseases 10th Revision) criteria based on audiometric examination.

\section{DNA extraction}

Genomic DNA from eight subjects in the HBJ family and 531 Han Chinese with normal hearing was extracted from peripheral blood leukocytes using a blood DNA extraction kit (Qiagen, Hilden, Germany), in accordance with the manufacturer's instructions. Ultraviolet spectrophotometry was used to measure the DNA concentration and purity.

\section{Screening for mutations in common deafness-related genes}

Screening for mutations in common deafness-related genes was conducted using polymerase chain reaction (PCR) amplification and direct sequencing of exons. These included GJB2, SLC26A4, and mitochondrial $12 S$ $r R N A$; the primers and PCR conditions were as 


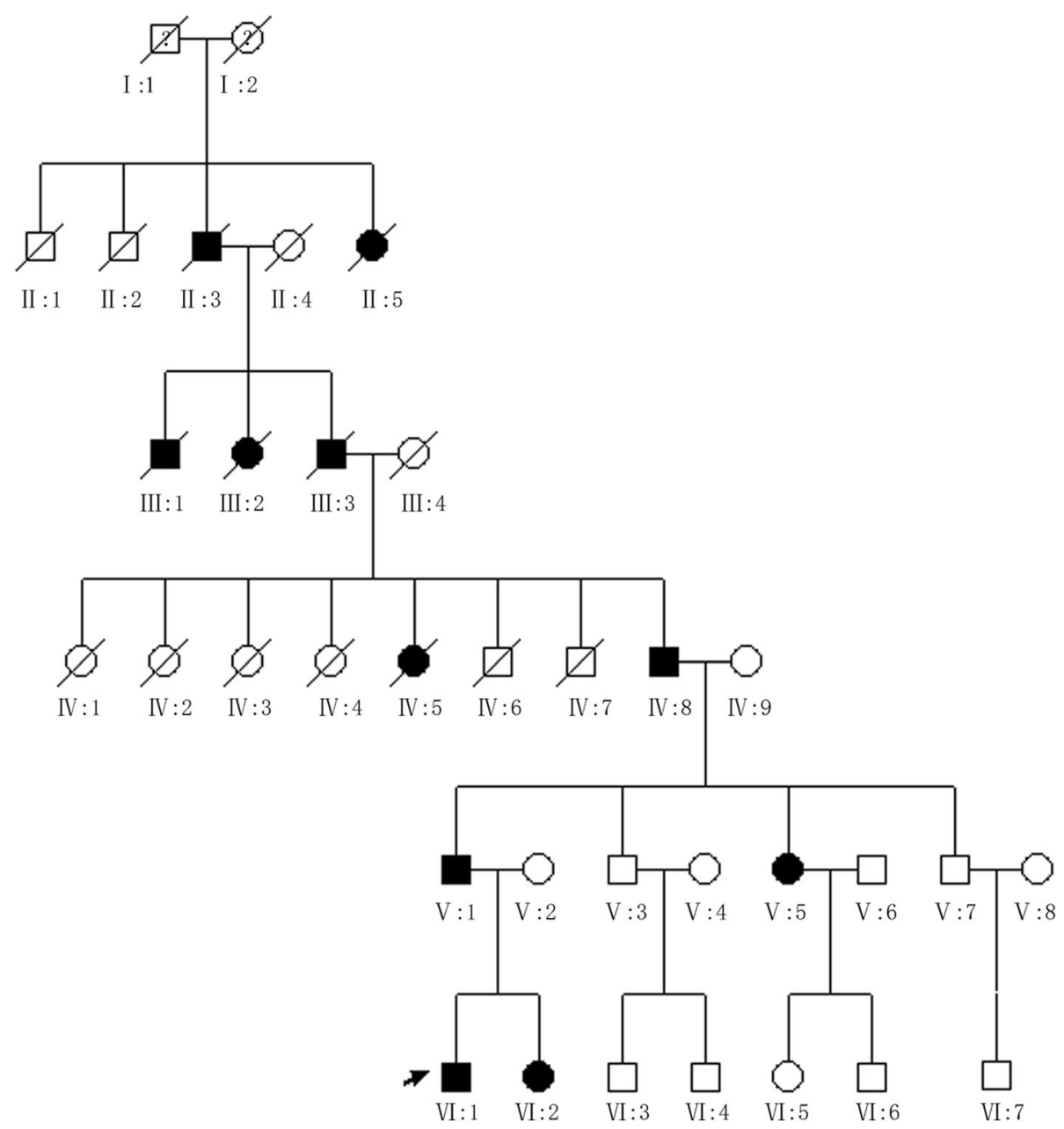

Fig. 1 Pedigree of the Chinese DFNA family HBJ. Affected family members are denoted in black. The arrow indicates the proband

described in detail in our previous paper [8]. The results of screening of these common deafness-related genes were all negative.

\section{Deafness gene capture and Illumina library preparation}

Mutation screening of 129 genes associated with hearing loss was performed in five family members by NGS. Deafness genes capture and Illumina library preparation were performed as the description in our previous paper $[9,10]$.

\section{Sanger sequencing}

After filtering against multiple databases, Sanger sequencing was used to determine whether any of the potential mutations in known genes causing ADNSHI cosegregated with the phenotype in this family. Direct PCR products were sequenced using Bigdye terminator v3.1 cycle sequencing kits (Applied Biosystems, Foster City, CA, USA) and analysed using an ABI 3700XL Genetic Analyzer.

\section{Mutational analysis}

Segregation of the mutations was evaluated in the family. Genotyping for c.887G > A was performed by PCR and detected by bidirectional sequencing of the amplified fragments using an automated DNA sequencer (ABI3100); the primers were $5^{\prime}$-GAATCCATCTATGA CCCTAACCA-3' and 5' - GCTTCTCGAAGTGCTTCT GC-3'. Nucleotide alterations were identified by sequence alignment with the KCNQ4 GenBank sequence (NM_014208) using Genetool software.

\section{Multiple sequence alignment}

Multiple sequence alignment was performed across 15 species using Clustal Omega (http://www.ebi.ac.uk/ Tools/msa/clustalo/).

\section{Model building and structure-based analysis}

Three-dimensional modeling of the human wild-type and p.G296D mutant of KCNQ4 was performed using SWISSMODEL. In this study, the automatic modeling 


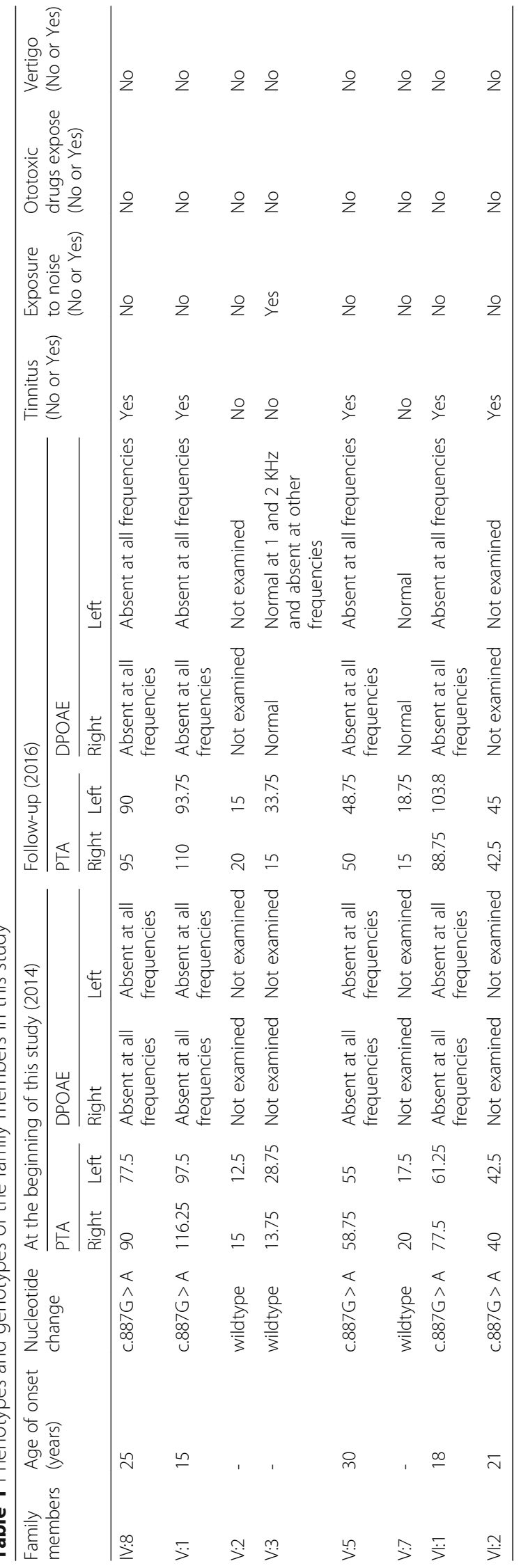


approach was applied to the complete protein sequence of human KCNQ4, including its 695 amino acids, and its mutant, which are available in NCBI GenBank (NP_004691.2) in FASTA format. Data obtained by the homology models were visualized using Swiss-PdbViewer 4.1.

\section{Results}

\section{Clinical evaluations}

In the HBJ family, 5 clinically affected and 12 unaffected individuals were identified. The age at onset of hearing impairment ranged from 15 to 30 years. The audiological assessments and clinical history of the affected members in this family showed postlingual, progressive, symmetrical, bilateral, non-syndromic, sensorineural hearing loss. The audiogram patterns of the patients were distinct; most of them initially showed high-frequency hearing loss, but in the proband (VI:1), hearing loss at all frequencies was seen from the outset. The hearing loss initially involved high frequencies, with subsequent gradual progression to a severe level involving all frequencies. The subjects with hearing loss also reported tinnitus, but there were no vestibular signs or symptoms (Table 1, Fig. 2).

An analysis of the temporal bone scans of the affected members by HRCT showed a normal middle-ear structure, including normal internal auditory canal and vestibular aqueduct. DPOAE testing showed cochlear dysfunction in the patients.

\section{Candidate gene analyses}

After the filtering process, we found that variants in MYO7A, GJB2, MITF, DIAPH1, PDZD7, WFS1, TRIOBP, GPR98, MYO1A, MYO15A and KCNQ4 might be the potential mutations. For details please see Additional file 1: Table S1. However, only the variant in KCNQ4 co-segregated within the family. We identified a novel mutation [c.887G $>$ A (p.G296D)] in exon 6 of KCNQ4 in the five affected family members. This mutation results in a glycine to aspartic acid substitution at position 296 in KCNQ4. Sanger sequencing revealed that all of the affected family members were heterozygous for this mutation, which was not present in the unaffected family members (Fig. $3 \mathrm{a} \sim \mathrm{h}$ ). The KCNQ4 c.887G > A mutation was also not detected in the normal hearing controls. The depth and coverage information for the DFNA2 locus was provided in Additional file 2: Table S2.

The glycine at position 296 is conserved across 15 species, as depicted in Fig. 3i. PROVAN, SIFT, PolyPhen, Mutation Taster and MutationAssessor predicted that KCNQ4 c.887G > A (p.G296D) would be a detrimental mutation (Table 2). In terms of protein structure, glycine is highly conserved in the $\mathrm{K}^{+}$channels of 15 different species, and is completely conserved among members of the KCNQ family, as well as of the super-family of voltagegating $\mathrm{K}^{+}$channels.

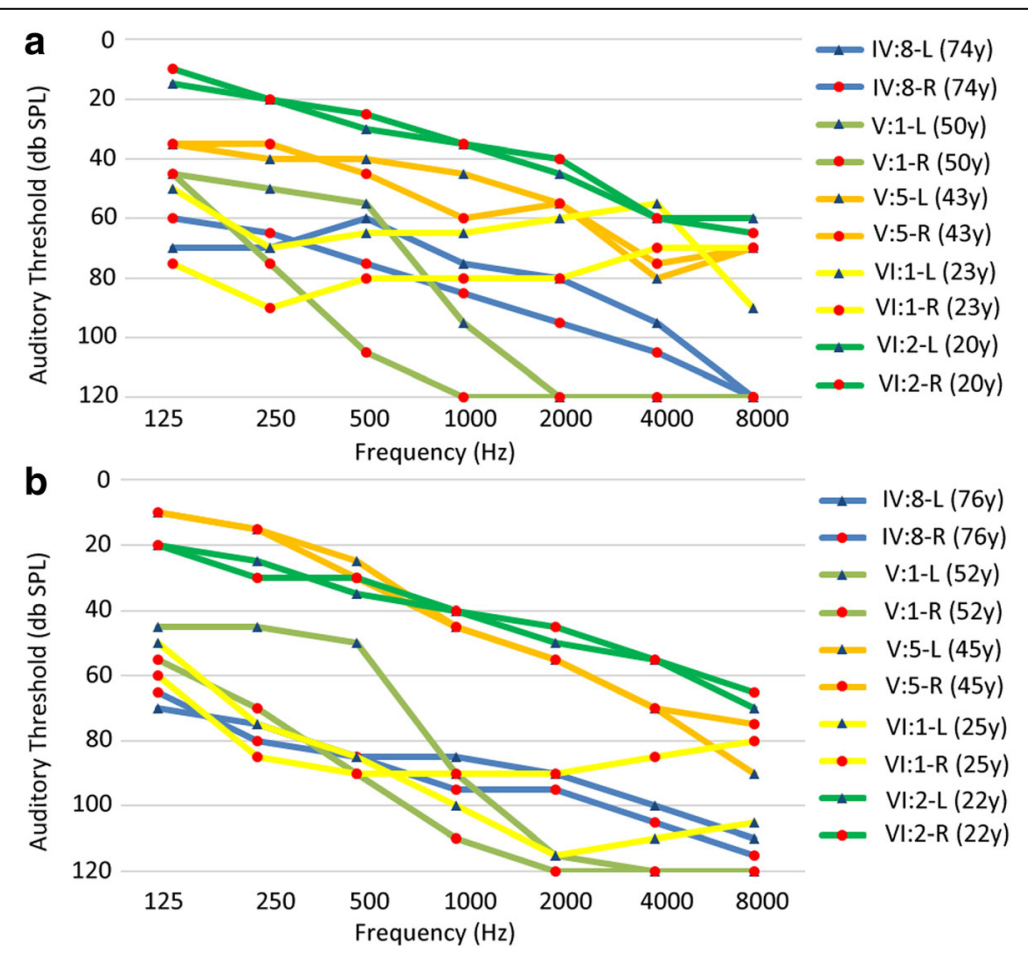

Fig. 2 The audiometric curves of the affected members in HBJ. a At the beginning of the study in 2014 ; b Follow up in 2016 


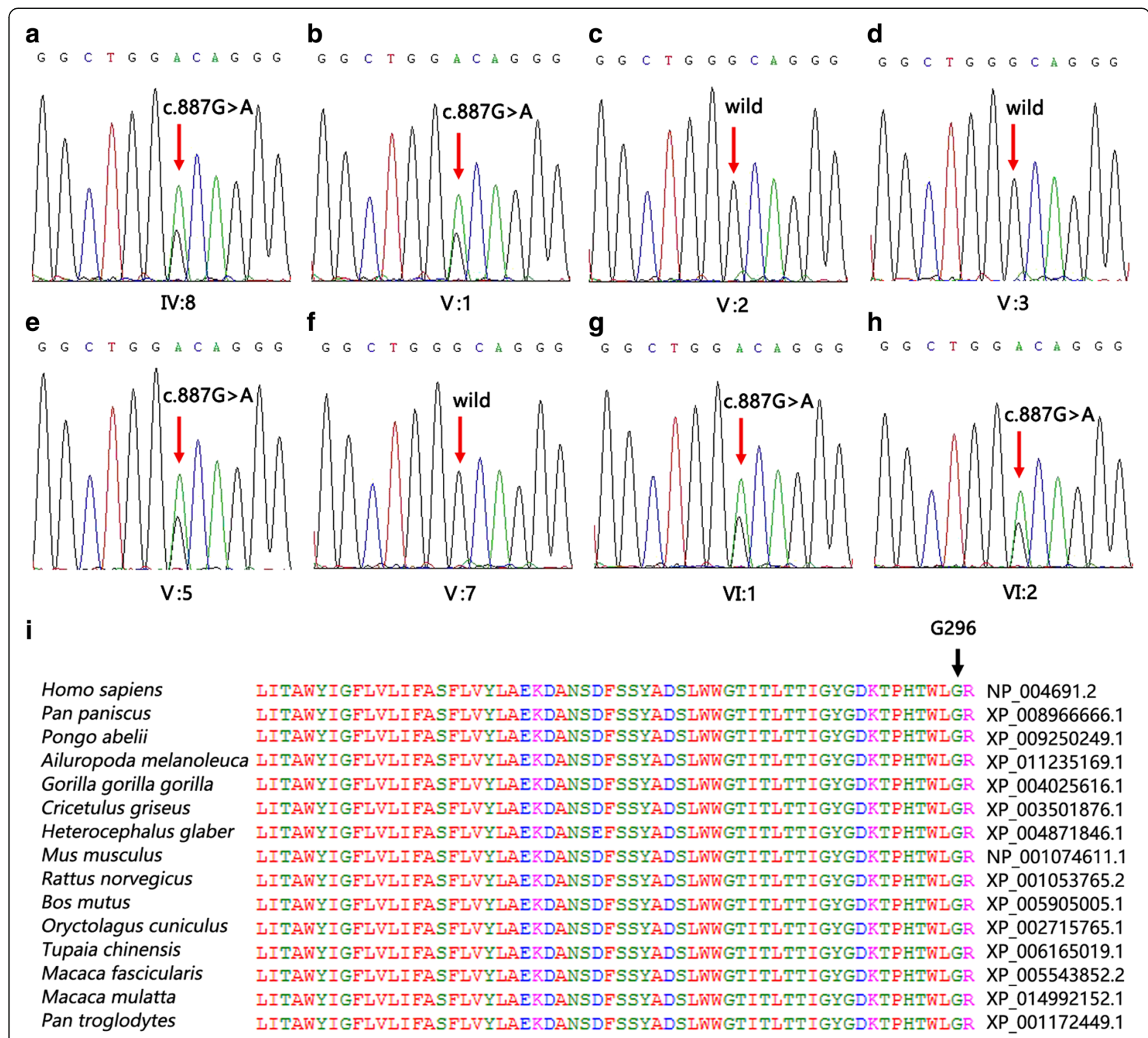

Fig. 3 Mutation detection and conservation analysis. $\mathbf{a} \sim \mathbf{h}$ The sanger sequence results of the family members; $\mathbf{i}$ Protein alignment shows conservation of the G296 residue of KNCQ4 across 15 species

\section{Structural modeling of p.G296D}

A structural model of the p.G296D variant of KCNQ4 was constructed based on the crystal structure of 4chvA. The model covered the target

Table 2 Pathogenicity Assessment in Silico of KCNQ4 c.887G > A (p.G296D)

\begin{tabular}{lll}
\hline Tools & Pathogenicity & $\begin{array}{l}\text { Functional Prediction Scores/ } \\
\text { Conservation scores }\end{array}$ \\
\hline PROVEAN & Deleterious & -6.558 \\
SIFT & Damaging & 0 \\
PolyPhen & Probably damaging & 1 \\
Mutation Taster & Disease causing & 1 \\
MutationAssessor & & 4.39 \\
\hline
\end{tabular}

sequence of KCNQ4 (residues 101-335). SwissPdbViewer 4.1 predicted that this mutation perturbs the amino acid side chain because of the replacement of glycine by aspartic acid. Both wild-type Glycine and mutant-type Aspartic forms two salt bridges with Leucine at position 299 and Alanine at position 300 (Fig. 4). Since the sequence identity between the target and the template was only $22.46 \%$, there might be other protein structure change that were absent in this model.

\section{Discussion}

The KCNQ4 gene encodes potassium voltage-gated channel subfamily KQT member 4, which is 

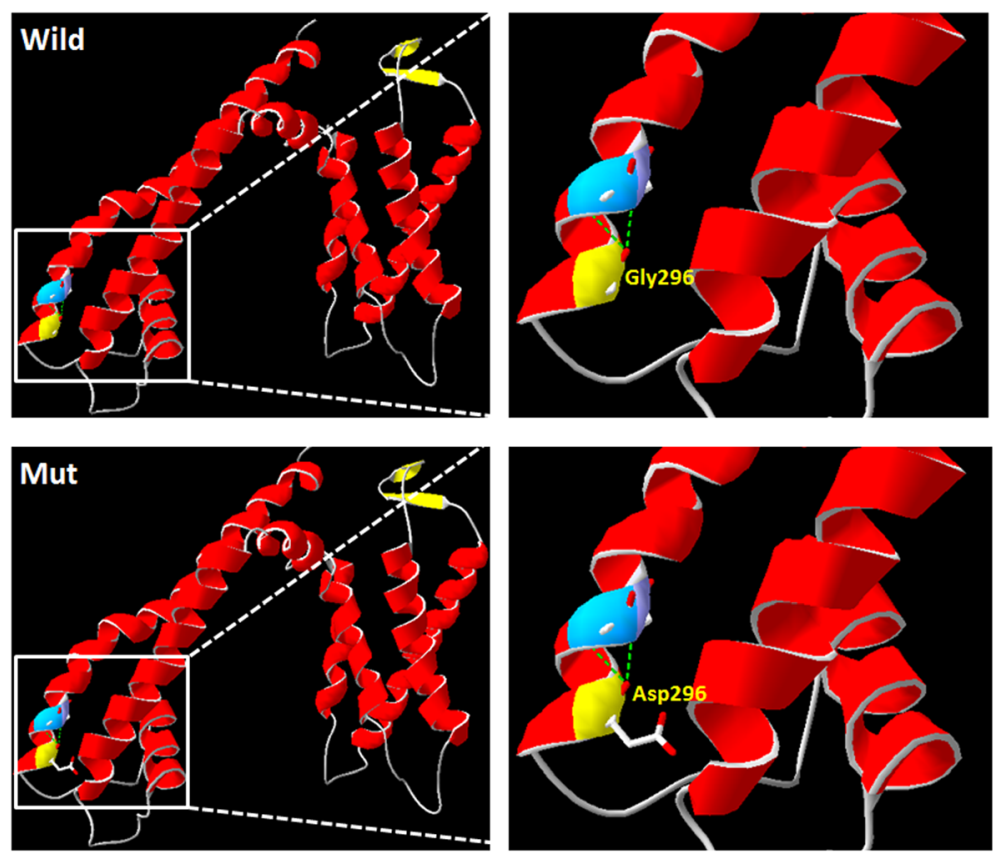

Fig. 4 Structure of wild-type and mutant 296 of KCNQ4. Upper panel: wild-type G296; lower panel: mutant D296

expressed in sensory outer hair cells (OHCs) [4]. KCNQ4 is linked to the DFNA2 locus on chromosome $1 \mathrm{p} 34$, which is thought to play a critical role in the regulation of neuronal excitability, particularly in sensory cells of the cochlea. Mutations in KCNQ4 have been shown to be associated with ADNSHL, mainly by impairing the function of OHCs [4]. To date, 20 mutations in $K C N Q 4$ have been reported to cause hearing loss. All are located in exons 1, 4-8 of KCNQ4 (Table 3) [4, 7, 11-25].

According to previous reports, mutations in KCNQ4 exhibit autosomal dominant inheritance. However, Wasano et al. recently identified another novel KCNQ4 mutation, c.1044_1051del8 (p.A349Pfs), in a family with autosomal recessive NSHL [25]. This suggests that mutation in $\mathrm{KCNQ} 4$ can cause autosomal recessive hearing loss with a more severe phenotype than autosomal dominant hearing loss.

Using NGS as well as Sanger sequencing, we identified a novel mutation, c.887G > A (p.G296D), which results in a glycine-to-aspartic acid substitution at position 296 in the pore region of KCNQ4. This mutation cosegregated with the phenotype in the family and was not detected in normal hearing controls.

The novel missense mutation p.G296D is in the pore region of $\mathrm{KCNQ} 4$, where also resides the mutations p.L274H [23], p.W275R [24], p.W276S [12, 15, 21, 22], p.L281S [20], p.G285S [4, 24], p.G285C [4], p.G287R [13], p.P291S [18], p.P291L [18] and p.G296S [7]. In a previous study, Mencia et al. reported that the mutation
c.886G > A (p.G296S) in KCNQ4 is pathogenic [7]. Mencia et al. demonstrated both effects of the mutation: reduced surface expression and abolished channel function. We speculate that the p.G296D mutation may affect the function of KCNQ4 channel through the mechanism that the mutation of amino acid residues in a single subunit of a tetrameric channel can block the permeation pathway and inhibit the current, leading to depolarization and death of outer hair cells $[4,6,26]$.

The age of onset of hearing loss, which ranges from the first to the fifth decade of life, differs among those with different mutations in KCNQ4. The age of onset of hearing loss caused by p.Q71fs in the N-terminal, p.G285C in the pore region, and p.A349Pfs in S6trans is in the first decade of life $[15,23,25]$. However, the average age of onset of hearing loss associated with the mutations p.W275R, p.G285S and p.G296S, located in the pore region, ranges from the first to the fourth decade $[4,7,24]$. In our study, the age of onset of hearing loss in the affected family members was in the second to third decades of life. In addition, the affected family members showed different hearing phenotypes. Most of the patients initially showed high-frequency hearing loss, while in the proband (VI-1), hearing of all frequencies was affected from the outset. This suggests that the same mutation in the same gene may have different effects among individuals in the same family. The present report, which is the first of p.G296D mutation in KCNQ4, adds to our understanding of KCNQ4 
Table 3 Overview of KCNQ4 mutations described in DFNA2

\begin{tabular}{|c|c|c|c|c|c|c|}
\hline Mutation & Protein change & Exon & Origin & Age of onset & Sub-domain & Reference \\
\hline c.211delC & p.Q71fs & 1 & Japan & $8-50$ & N-term cyto & {$[17,18]$} \\
\hline c.211del13 & p.Q71fs & 1 & Belgian & $<10$ & N-term cyto & [15] \\
\hline c.229_230insGC & p.H77fs & 1 & Japan & 27 & N-term cyto & [18] \\
\hline$c .546 C>$ G & p.F182L & 4 & Japan & - & S3 trans & {$[18,19]$} \\
\hline c.664_681del18 & p.G215_220del6 & 4 & Korea & earliest 4 & S4-S5 linker & [14] \\
\hline c.689 T > A & p.V230E & 4 & Japan & $3-40$ & S4-S5 linker & [18] \\
\hline$c .725 G>A$ & p.W241X & 5 & America & - & S5 trans & [16] \\
\hline c.778G > A & p.E260K & 5 & America & - & S5 trans & [16] \\
\hline c.785A > T & p.D262V & 5 & America & - & S5 trans & [16] \\
\hline c.806_808delCCT & p.S269del & 5 & Canada & $<10$ & S5-S6 linker & [11] \\
\hline$c .821 \mathrm{~T}>\mathrm{A}$ & p.L274H & 5 & Neth & - & $\mathrm{PR}(\mathrm{P})$ & [23] \\
\hline c.823 T > C & p.W275R & 5 & China & $2-30$ & $\mathrm{PR}(\mathrm{P})$ & [24] \\
\hline$c .827 G>C$ & p.W276S & 5 & Japan,Dutch & $<10$ & $\mathrm{PR}(\mathrm{P})$ & {$[12,15,21,22]$} \\
\hline $\mathrm{c} .842 \mathrm{~T}>\mathrm{C}$ & p.L281S & 6 & America & - & $\mathrm{PR}(\mathrm{P})$ & [20] \\
\hline$c .853 G>T$ & p.G285C & 6 & America & $<10$ & $\mathrm{PR}(\mathrm{P})$ & [15] \\
\hline$c .853 G>A$ & p.G285S & 6 & French,China & $6-30$ & $\mathrm{PR}(\mathrm{P})$ & {$[4,24]$} \\
\hline$c .859 G>C$ & p.G287R & 6 & America & $1-21$ & $\mathrm{PR}(\mathrm{P})$ & [13] \\
\hline$c .871 C>T$ & p.P291S & 6 & Japan & 20 & $P R(P)$ & [18] \\
\hline c. $872 C>T$ & p.P291L & 6 & Japan & 17 & $P R(P)$ & [18] \\
\hline$c .886 G>A$ & p.296S & 6 & Spanish & $9-50$ & $P R$ & [7] \\
\hline $\mathrm{c} .887 \mathrm{G}>\mathrm{A}$ & p.G296D & 6 & China & $15-25$ & $P R$ & This study \\
\hline$c .891 G>T$ & p.R297S & 6 & Japan & 5,39 & S6 trans & [18] \\
\hline$c .961 G>A$ & p.G321S & 7 & Dutch & $<10$ & S6 trans & [15] \\
\hline c.1044_1051del8 & p.A349Pfs & 8 & Japan & $<10$ & S6-B segment linker & [25] \\
\hline
\end{tabular}

Abbreviations: cyto cytoplasmic, trans transmembrane, $P R$ Pore region, (P) P-loop

mutation-induced hearing loss. This study also indicates that NGS is a valuable tool for the diagnosis of autosomal dominant deafness. We plan to follow the next generation of this family and hope to obtain further valuable information from them. In addition, we hope to provide them with more comprehensive genetic counseling, early diagnosis, and even treatment of hearing impairment.

\section{Conclusions}

In this study, a novel KCNQ4 mutation, c. $887 \mathrm{G}>\mathrm{A}$ (p.G296D), was identified in all five affected members in a Chinese family with ADNSHL using NGS and Sanger sequencing. The hearing phenotype of the proband differed from that in previously reported pedigrees, and in other members of the same family with KCNQ4 mutations. The same mutation in the same gene may thus have different effects among individuals in the same family. This may be related to the expressivity of the gene and the age of onset in the autosomal hereditary mode. The results of our study increase the content of databases of genes associated with genetic deafness.

\section{Additional files}

Additional file 1: Table S1. Potential deafness causing variants found by NGS. (DOCX 14 kb)

Additional file 2: Table S2. Depth and coverage information of DFNA2 locus in NGS. (DOCX 14 kb)

\section{Abbreviations}

ADNSHL: Autosomal dominant non-syndromic hearing loss; DFNA: Non-syndromic deafness autosomal dominant; DPOAE: Distortion product otoacoustic emissions; gDNA: Genomic DNA; HRCT: High-resolution computed tomography; NGS: Next-generation sequencing; NSHL: Non-syndromic hearing loss; PCR: Polymerase chain reaction; PTA: Pure tone audiometry

\section{Acknowledgements}

We sincerely thank all the family members for their participation and cooperation in this study. 


\section{Funding}

These investigations were supported by grant from National Key Research and Development Project (2016YFC1000706),National Natural Science Foundation of China (81371098) to Yongyi Yuan and National Key Research and Development Project (2016YFC1000700, 2016YFC1000704),National Natural Science Foundation of China $(81230020,81371096)$ to Pu Dai. National Natural Science Foundation of China (81570929) to Xue Gao. Natural Science Foundation of Hebei Province of China (C2010000571) and Medical applicable Technical Track Project of Hebei Province of China (GL2011-35) to Qingwen Zhu. The funders had no role in study design, data collection and analysis, decision to publish, or preparation of the manuscript.

\section{Availability of data and materials}

The datasets generated and/or analyzed during the current study are available from the corresponding author on reasonable request.

\section{Authors' contributions}

$\mathrm{BH}, \mathrm{YL}$ and $\mathrm{XG}$ carried out the molecular genetic studies and sequence alignment. $\mathrm{BH}, \mathrm{JX}$ and $\mathrm{YL}$, carried out the bioinformatic analysis of the sequencing data. $Y Y$ and $B H$ drafted the manuscript. $Y Y, Q Z$ and PD conceived the study, participated in its design and coordination. All authors have read and approved the final manuscript.

\section{Competing interests}

The authors declare that they have no competing interests.

\section{Consent for publication}

Not applicable.

\section{Ethics approval and consent to participate}

This study was approved by the Chinese PLA General Hospital Research Ethics Committee and the Ethical Review Committee of the Second Hospital of Hebei Medical University, and informed consent had been obtained from all of the participants, including the parents of minors.

\section{Publisher's Note}

Springer Nature remains neutral with regard to jurisdictional claims in published maps and institutional affiliations.

\section{Author details}

'Department of Otolaryngology, Chinese PLA General Hospital, Beijing 100853, China. ${ }^{2}$ Department of Otolaryngology, Hainan Branch of PLA General Hospital, Sanya 572013, China. ${ }^{3}$ Department of Otorhinolaryngology, General Hospital of the Rocket Force, Beijing 100088, China. ${ }^{4}$ Department of Otolaryngology, The Second Hospital Of Hebei Medical University, Shijiazhuang 050018, China.

\section{Received: 16 August 2016 Accepted: 8 March 2017}

\section{Published online: 24 March 2017}

\section{References}

1. Morton CC, Nance WE. Newborn hearing screening-a silent revolution. N Engl J Med. 2006;354(20):2151-64.

2. Baek Jl, Oh SK, Kim DB, Choi SY, Kim UK, Lee KY, Lee SH. Targeted massive parallel sequencing: the effective detection of novel causative mutations associated with hearing loss in small families. Orphanet J Rare Dis. 2012;7:60.

3. Hilgert N, Smith RJ, Van Camp G. Forty-six genes causing nonsyndromic hearing impairment: which ones should be analyzed in DNA diagnostics? Mutat Res. 2009;681(2-3):189-96.

4. Kubisch C, Schroeder BC, Friedrich T, Lutjohann B, El-Amraoui A, Marlin S, Petit C, Jentsch TJ. KCNQ4, a novel potassium channel expressed in sensory outer hair cells, is mutated in dominant deafness. Cell. 1999; 96(3):437-46.

5. Doyle DA, Morais Cabral J, Pfuetzner RA, Kuo A, Gulbis JM, Cohen SL, Chait BT, Mackinnon R. The structure of the potassium channel: molecular basis of K+ conduction and selectivity. Science. 1998;280(5360):69-77.

6. Kharkovets T, Dedek K, Maier H, Schweizer M, Khimich D, Nouvian R, Vardanyan V, Leuwer R, Moser T, Jentsch TJ. Mice with altered KCNQ4 K+ channels implicate sensory outer hair cells in human progressive deafness. EMBO J. 2006;25(3):642-52.
7. Mencia A, Gonzalez-Nieto D, Modamio-Hoybjor S, Etxeberria A, Aranguez G, Salvador N, Del Castillo I, Villarroel A, Moreno F, Barrio L, et al. A novel KCNQ4 pore-region mutation (p.G296S) causes deafness by impairing cell-surface channel expression. Hum Genet. 2008;123(1): $41-53$.

8. Xin F, Yuan Y, Deng X, Han M, Wang G, Zhao J, Gao X, Liu J, Yu F, Han D, et al. Genetic mutations in nonsyndromic deafness patients of Chinese minority and Han ethnicities in Yunnan, China. J Transl Med. 2013;11:312.

9. Huang A, Yuan Y, Liu Y, Zhu Q, Dai P. A novel EYA4 mutation causing hearing loss in a Chinese DFNA family and genotype-phenotype review of EYA4 in deafness. J Transl Med. 2015;13:154.

10. Yuan Y, Gao X, Huang B, Lu J, Wang G, Lin X, Qu Y, Dai P. Phenotypic Heterogeneity in a DFNA20/26 family segregating a novel ACTG1 mutation. BMC Genet. 2016;17:33.

11. Abdelfatah N, McComiskey DA, Doucette L, Griffin A, Moore SJ, Negrijn C, Hodgkinson KA, King JJ, Larijani M, Houston J, et al. Identification of a novel in-frame deletion in KCNQ4 (DFNA2A) and evidence of multiple phenocopies of unknown origin in a family with ADSNHL. Eur J Hum Genet. 2013;21(10):1112-9.

12. Akita J, Abe S, Shinkawa H, Kimberling WJ, Usami S. Clinical and genetic features of nonsyndromic autosomal dominant sensorineural hearing loss: KCNQ4 is a gene responsible in Japanese. J Hum Genet. 2001;46(7):355-61

13. Arnett J, Emery SB, Kim TB, Boerst AK, Lee K, Leal SM, Lesperance MM. Autosomal dominant progressive sensorineural hearing loss due to a novel mutation in the KCNQ4 gene. Arch Otolaryngol Head Neck Surg. 2011; 137(1):54-9.

14. Baek Jl, Park HJ, Park K, Choi SJ, Lee KY, Yi JH, Friedman TB, Drayna D, Shin KS, Kim UK. Pathogenic effects of a novel mutation (c.664_681del) in KCNQ4 channels associated with auditory pathology. Biochim Biophys Acta. 2011; 1812(4):536-43.

15. Coucke PJ, Van Hauwe P, Kelley PM, Kunst H, Schatteman I, Van Velzen D, Meyers J, Ensink RJ, Verstreken M, Declau F, et al. Mutations in the KCNQ4 gene are responsible for autosomal dominant deafness in four DFNA2 families. Hum Mol Genet. 1999:8(7):1321-8.

16. Hildebrand MS, Tack D, McMordie SJ, DeLuca A, Hur IA, Nishimura C, Huygen P, Casavant TL, Smith RJ. Audioprofile-directed screening identifies novel mutations in KCNQ4 causing hearing loss at the DFNA2 locus. Genet Med. 2008:10(11):797-804.

17. Kamada F, Kure S, Kudo T, Suzuki Y, Oshima T, Ichinohe A, Kojima K, Niihori T, Kanno J, Narumi Y, et al. A novel KCNQ4 one-base deletion in a large pedigree with hearing loss: implication for the genotype-phenotype correlation. J Hum Genet. 2006;51(5):455-60.

18. Naito T, Nishio SY, Iwasa Y, Yano T, Kumakawa K, Abe S, Ishikawa K, Kojima H, Namba A, Oshikawa C, et al. Comprehensive genetic screening of KCNQ4 in a large autosomal dominant nonsyndromic hearing loss cohort: genotype-phenotype correlations and a founder mutation. PLoS One. 2013:8(5):e63231.

19. Su CC, Yang JJ, Shieh JC, Su MC, Li SY. Identification of novel mutations in the KCNQ4 gene of patients with nonsyndromic deafness from Taiwan. Audiol Neurootol. 2007;12(1):20-6.

20. Talebizadeh Z, Kelley PM, Askew JW, Beisel KW, Smith SD. Novel mutation in the KCNQ4 gene in a large kindred with dominant progressive hearing loss. Hum Mutat. 1999:14(6):493-501.

21. Topsakal V, Pennings RJ, te Brinke $H$, Hamel B, Huygen PL, Kremer $H$, Cremers $\mathrm{CW}$. Phenotype determination guides swift genotyping of a DFNA2/KCNQ4 family with a hot spot mutation (W276S). Otol Neurotol. 2005;26(1):52-8

22. Van Camp G, Coucke PJ, Akita J, Fransen E, Abe S, De Leenheer EM, Huygen $\mathrm{PL}$, Cremers CW, Usami S. A mutational hot spot in the KCNQ4 gene responsible for autosomal dominant hearing impairment. Hum Mutat. 2002; 20(1):15-9.

23. Van Hauwe P, Coucke PJ, Ensink RJ, Huygen P, Cremers CW, Van Camp G. Mutations in the KCNQ4 K+ channel gene, responsible for autosomal dominant hearing loss, cluster in the channel pore region. Am J Med Genet. 2000;93(3):184-7.

24. Wang H, Zhao Y, Yi Y, Gao Y, Liu Q, Wang D, Li Q, Lan L, Li N, Guan J, et al. Targeted high-throughput sequencing identifies pathogenic mutations in KCNQ4 in two large Chinese families with autosomal dominant hearing loss. PLoS One. 2014;9(8):e103133. 
25. Wasano K, Mutai H, Obuchi C, Masuda S, Matsunaga T. A novel frameshift mutation in KCNQ4 in a family with autosomal recessive non-syndromic hearing loss. Biochem Biophys Res Commun. 2015; 463(4):582-6.

26. Holt JR, Stauffer EA, Abraham D, Geleoc GS. Dominant-negative inhibition of M-like potassium conductances in hair cells of the mouse inner ear. J Neurosci. 2007;27(33):8940-51. 